\title{
Elementos de una propuesta metodológica para el desarrollo de la Responsabilidad Social basada en la norma ISO 26000:2010, en una IPS de tercer nivel de complejidad en la ciudad de Bogotá*
}

Elements of a methodology development based Social Responsibility ISO 26000:2010, in a third IPS complexity level the city of Bogotá

Hermelinda Gómex Ramírez"*

Policía Nacional

Eliana Carolina Herrera Pérez"**

Neumo Investigaciones SAS

Ligia Edilma Sorro Corredor"***

Comunidad Hermanas Dominicas de la Presentación

\footnotetext{
Artículo de resultado de investigación.

** Fonoaudióloga. Especialista en Audiología. Magíster (c) en Calidad y Gestión Integral. Policía Nacional. Correo electrónico: fonomely@hotmail.com

*** Química farmacéutica. Magíster (c) en Calidad y Gestión Integral. Neumo Investigaciones SAS. Correo electrónico: ecarolinaherrera@hotmail.es

**** Licenciada en Ciencias Religiosas. Magíster en Gestión Social Empresarial. Magíster (c) en Calidad y Gestión Integral. Pertenece a la comunidad Hermanas Dominicas de la Presentación. Correo electrónico: ligiasorro211@hotmail.com
} 


\section{RESUMEN}

La Responsabilidad Social (RS) en Colombia ha tenido poca incorporación en el sector servicios, el cual ha tenido un amplio crecimiento en las últimas décadas. El sector de la salud es uno de los más afectados en cuanto al componente humano debido a los efectos de la globalización, la cual ha generado deshumanización en la prestación de los servicios, situación que evidencia la necesidad de vincular la Responsabilidad Social en la gestión de las IPS. Este artículo presenta los resultados de una investigación que buscó determinar los elementos de una metodología para facilitar el desarrollo de la $\mathrm{RS}$ basada en los requisitos de la norma ISO 26000:2010 - Guía de Responsabilidad Social, a partir del diagnóstico de las prácticas de RS en una IPS de tercer nivel de complejidad en la ciudad de Bogotá.

Se elaboró un instrumento para el diagnóstico de las prácticas de RS en la IPS, que fue sometido a revisión técnica y a prueba piloto $(\mathrm{n}=33)$ previa, el cual fue aplicado a las partes interesadas ( $n=164)$ de la institución. Los resultados del diagnóstico fueron priorizados y a partir de su triangulación con la información derivada de la revisión de literatura y la lluvia de ideas del equipo de investigadores, se definieron las alternativas de intervención y se elaboró una propuesta de metodología para el desarrollo de la RS basada en la norma en mención y en el informe de sostenibilidad GRI-4, para facilitar la adopción de prácticas de RS alineadas con los requisitos del Sistema Obligatorio de Garantía de Calidad SOGCS, para fomentar una cultura de RS y contribuir en la prestación de servicios de calidad y humanizados.

Palabras clave: instituciones prestadoras de servicios de salud, Responsabilidad Social, norma ISO 26000:2010 - Guía de Responsabilidad Social, desarrollo sostenible.

\section{ABSTRACT}

Social Responsibility (SR) in Colombia has been incorporated little in the service sector, which has had a large growth in recent decades. Health sector is one of the most affected in their human component due to the effects of globalization, which have generated dehumanization in the provision of services, which highlights the need to link social responsibility in the management of IPS. This article presents the results of a study that sought to determine the elements of a methodology for the development of the SR based on the ISO 26000:2010 - Guidance on Social Responsibility requirements and diagnosis of SR practices in third-level complexity IPS of Bogotá. It corresponds to a research mixed with multiple integration.

An instrument was developed for the diagnosis of SR practices in the IPS, which was subjected to technical review and test pilot $(n=33)$ prior to their application, which was applied to stakeholders ( $\mathrm{n}=164$ ) of was prepared institution. Diagnostic results were prioritized and triangulated with information derived from the literature review and brainstorming team of researchers alternatives intervention were defined and a proposed methodology for the development of the SR based on the Standard was developed in mention and sustainability report GRI-4, to facilitate the adoption of SR practices aligned with SOGCS requirements, to promote a culture of SR and contribute in providing quality and humanized services.

Keywords: Service institutions health, Social Responsibility, ISO 26000:2010 - Guidance on Social Responsibility, sustainable development. 


\section{INTRODUCCIÓN}

La Responsabilidad Social (RS) surgió en los años 50 y se asoció al rápido incremento del tamaño y poder de las empresas americanas, y al papel protagónico que estas organizaciones tenían en una sociedad que afrontaba problemas sociales (inequidad, contaminación, pobreza, entre otros); en este sentido, la RS en las organizaciones se convirtió en una exigencia compartida por diversos grupos que solicitaban cambios en los negocios americanos (González de la Cuesta, 2005, p. 33).

La RS ha tenido un largo desarrollo conceptual; sin embargo, en síntesis la introducción de la RS en las estrategias organizacionales se debe considerar como una inversión que brinda ventajas competitivas y que agrega valor a la visibilidad de la organización, debido a sus aportes en la generación de programas orientados a la reducción de la inequidad socioeconómica de las poblaciones, así como en la minimización del impacto ambiental de sus acciones.

Cabe destacar, que como un reflejo de la importancia que fue adquiriendo en el ámbito mundial, la International Organization for Standardization (ISO) en el 2004 dio inicio al proceso de desarrollo de una guía sobre Responsabilidad Social, el cual concluyó en noviembre de 2010 con la publicación de la norma ISO 26000.

El desarrollo de esta norma se constituyó en el mayor proceso de convergencia en torno a la RS, puesto que incluyó a más de 90 países y 50 organizaciones internacionales. Además, en su construcción participaron diversas categorías de partes interesadas y se tuvo representatividad de los países en desarrollo como Brasil y Sudáfrica.

En la norma ISO 26000:2010 la RS se concibe como:

La responsabilidad de una organización ante los impactos que sus decisiones y actividades ocasionan en la sociedad y el medio ambiente, mediante un comportamiento ético y transparente que: i. Contribuya al desarrollo sostenible, incluyendo la salud y el bienestar de la sociedad; ii. Tome en consideración los intereses de sus partes interesadas; iii. Cumpla con la legislación aplicable y sea coherente con la normativa internacional de comportamiento; y iv. Esté integrada en toda la organización y se lleve a la práctica en sus relaciones (Instituto Colombiano de Normalización y Certificación, Icontec, 2010).

En Colombia la RS se ha incorporado principalmente en organizaciones del sector manufacturero y muy poco en el sector de servicios, el cual ha tenido un amplio crecimiento en las últimas décadas; por consiguiente, se convierte en una oportunidad para la generación de nuevo conocimiento en este campo. Además, se encontró que el sector salud es uno de los más afectados en su componente humano, debido a los efectos que han traído consigo los procesos de globalización.

En este sentido, las condiciones de trabajo precarias (las cuales a su vez generan reducción en la motivación, el compromiso y la productividad de los profesionales en salud, así como alta rotación de personal, lo que interfiere en la continuidad de los procesos de intervención en salud y en su gestión) con las que cuentan actualmente los trabajadores de este sector, entre otros factores, han generado deshumanización en la prestación de servicios de salud, aspecto que sugiere la necesidad de vincular dentro de la gestión de estas instituciones estrategias de RS que faciliten la integración y el trabajo efectivo y humanizado de las IPS con las distintas comunidades que intervienen y sus familias.

Cabe precisar que las "personas construyen la realidad a partir de prácticas y discursos cargados de significados, desde los cuales orientan sus acciones" (Delgado, Vásquez y Moraes, 2016). Estos significados se crean a partir de la interacción con los otros, en este sentido, la prestación de los servicios de salud constituye una construcción social derivada de una interacción 
compleja entre diferentes actores sociales, y es aquí donde se requiere rescatar la RS como un mecanismo para la reducción de la deshumanización de los servicios en salud y de las barreras de acceso de las poblaciones a estos servicios, las cuales para el caso de Colombia son resultado de 4 factores: 1) el diseño de un sistema de salud segmentado con insuficiencia en la cobertura de servicios; 2) la inadecuada gestión de la salud y los mecanismos de acceso; 3) la estructura de las redes de atención y las limitaciones organizacionales; 4) las malas condiciones de vida de las poblaciones que se asocian a la pobreza de las mismas (Vargas, Vásquez, Mogollón, 2010; García, Vargas, Mogollón, De Paepe, Da Silva, Unger, Vázquez, 2014).

Por otra parte, al revisar la literatura se encontraron escasos estudios que vinculen la guía internacional ISO 26000:2010 de RS de forma integrada con el Sistema Obligatorio de Garantía de la Calidad en Salud SOGCS, y particularmente con el Sistema Único de Acreditación - SUA en la gestión de IPS en la ciudad de Bogotá, aspecto que evidencia un vacío en el conocimiento de este campo.

Además, teniendo en cuenta que las IPS son las encargadas de prestar los servicios en salud a las poblaciones, se hace necesaria la vinculación de estrategias de gestión de la RS, considerando estándares internacionales como la guía ISO 26000:2010, en la gestión global de la organización con el ánimo de contribuir a la prestación de servicios de salud con calidad y humanización.

Por otra parte, el desarrollo sostenible es una de las apuestas del siglo XXI del Programa de Naciones Unidas para el Desarrollo - PNUD, organización que ha aportado en la introducción de mejoras en la vida de las comunidades. El desarrollo sostenible en su definición simple se entiende como "las necesidades de la generación presente sin comprometer la capacidad de las generaciones futuras para satisfacer sus propias necesidades" (ONU, 2016). Consta de 3 pilares, los cuales se deben generar de manera equilibrada: el desarrollo económico, el desarrollo social y la protección del medio ambiente (Asamblea General de las Naciones Unidas, 2016). Desde una perspectiva global el desarrollo sostenible puede ser entendido como el "mantenimiento o el mejoramiento de las "condiciones de calidad" del sistema de interrelaciones sociedad-naturaleza" (Pérez, 2016).

Para el caso de Colombia se han adoptado los Objetivos de Desarrollo Sostenible - ODS, también conocidos como objetivos globales, los cuales son el resultado de un consenso global y fueron trazados en un periodo de 15 años, entre los años 2016 a 2030 (Programa de Naciones Unidas para el Desarrollo - PNUD, 2016).

Se espera que Colombia oriente políticas públicas y acciones dirigidas al cumplimiento de estos ODS. En ese sentido, este proyecto de investigación aporta en la generación de conocimiento para el cumplimiento de los objetivos específicos números 3 (salud y bienestar) y 8 (trabajo decente y crecimiento económico). En primer lugar, la IPS objeto de investigación, a través de la prestación de servicios de calidad y humanizados, contribuye en la garantía de una vida sana y promueve el bienestar de diferentes poblaciones a lo largo de su ciclo vital. En segundo lugar, al ser el sector salud uno de los más precarios en cuanto a condiciones de trabajo, se hace necesario promover políticas organizacionales orientadas por un lado al crecimiento económico sostenido, inclusivo y sostenible de la IPS, y por otro lado al empleo pleno y productivo y el trabajo decente para todos los profesionales de la salud y personal administrativo y de servicios vinculados a la institución. No obstante, para alcanzar estos propósitos se requiere de la incorporación de estrategias de RS que faciliten esta gestión y que visibilicen los adelantos de las IPS en $\mathrm{RS}$ entre sus diferentes grupos de interés.

Aunado a ello, el Global Reporting Initiative - GRI, es una organización no gubernamental basada en una 
red, que tiene como objetivo impulsar los reportes de sostenibilidad y de medio ambiente, social y gobierno corporativo. El GRI genera la estructura de reportes de sostenibilidad, los cuales son ampliamente utilizados en distintos países, debido a que son el resultado del consenso de distintos actores en diversos contextos (Global Reporting Initiative, 2016).

La estructura de reporte propuesta permite impulsar una mayor transparencia, debido a la estandarización de los criterios de evaluación y divulgación de las acciones emprendidas en este campo. El GRI proporciona orientaciones dirigidas a las organizaciones interesadas en reportar sobre los temas que aborda la norma ISO 26000 - Guía de Responsabilidad Social, además provee información detallada que da cuenta del contexto de sostenibilidad de las organizaciones y vincula elementos para el involucramiento de las partes interesadas en el proceso de reporte (Global Reporting Initiative, 2016).

Particularmente la IPS, objeto de la presente investigación, no cuenta con pautas de gestión que promuevan la RS como una estrategia para el fomento del desarrollo sostenible en las actividades desarrolladas por la institución. Así mismo, se detectó poca interacción social con las comunidades involucradas en el desarrollo de los proyectos, situación que evidencia la poca articulación de los principios y de las materias fundamentales de la RS en su gestión diaria.

De acuerdo con lo enunciado, se evidencia que la institución objeto de estudio no cuenta con una estructura metodológica debidamente definida que permita hacer un diagnóstico situacional de la organización frente a la gestión de la RS, así como no dispone de una metodología para la implementación de un sistema de gestión de RS que esté en línea los requerimientos que propone la guía ISO 26000:2010.

Este artículo muestra los resultados de una investigación cuyo objetivo fue determinar los elementos de una metodología para el desarrollo de la RS, basada en los requisitos de la norma ISO 26000:2010 - Guía de Responsabilidad Social a partir del diagnóstico del estado actual del desarrollo de la RS en una IPS de tercer nivel de complejidad en la ciudad de Bogotá.

\section{METODOLOGÍA}

Se desarrolló una investigación de tipo mixto con diseño de integración múltiple (Hernández, Collado y Baptista, 2014). Se elaboró un instrumento bajo criterios de rigor (Bernal, 2010, p. 342) para el diagnóstico de las prácticas de RS en la IPS basado en las materias fundamentales y en los principios de la norma ISO 26006:2010 - Guía de Responsabilidad Social, que fue sometido a una revisión técnica por parte de un médico con experiencia y formación en sistemas de gestión y en procesos de investigación y a una prueba piloto $(\mathrm{n}=33)$ previamente a su aplicación. La prueba piloto derivó ajustes y mejoras en la semántica y sintaxis de los enunciados propuestos como criterios a evaluar.

Este instrumento consta de 74 preguntas, de las cuales 40 corresponden a las 7 materias fundamentales y 34 a los 7 principios de la norma citada. Está dirigido a las partes interesadas, grupos de interés o stakeholders (usuarios y sus familias, colaboradores -profesionales de salud y personal administrativo-, proveedores, contratistas y junta de socios) de la IPS.

Las variables abordadas en la investigación corresponden a las materias fundamentales de las que da cuenta la RS, de acuerdo con lo establecido en la norma ISO 2006:2010 - Guía de Responsabilidad Social, a saber: 1) gobernanza de la organización; 2) derechos humanos; 3) prácticas laborales; 4) medio ambiente; 5) prácticas justas de operación (referidas a corrupción, competencia, cadena de valor); 6) asuntos de consumidores; 7) participación activa en la comunidad y desarrollo de 
la misma. Así mismo, se tomaron los 7 principios de la RS: 1) rendición de cuentas; 2) transparencia; 3) comportamiento ético; 4) respeto a los intereses de las partes interesadas; 5 ) respeto al principio de legalidad; 6) respeto a la normativa internacional de comportamiento; 7) respeto a los derechos humanos.

Una vez definidos los criterios de evaluación se definió la escala, y para ello se tomaron como base los niveles de evaluación propuestos en la matriz de autoevaluación (elementos claves y elementos detallados) del éxito sostenido que trae el anexo A de la norma ISO 9004:2009, los cuales se muestran en la tabla 1. La evaluación puede proporcionar una visión global de desempeño y del grado de madurez de la RS en la institución. También puede identificar áreas para la mejora y así determinar prioridades para acciones pertinentes.

Tabla 1. Niveles de desarrollo de la RS.

\begin{tabular}{|l|l|l|}
\hline \multicolumn{1}{|c|}{ Nivel de desarrollo } & \multicolumn{1}{c|}{ Descripción } \\
\hline 1 & Nivel de desarrollo nulo & La empresa no ha desarrollado ninguna iniciativa de esta práctica. \\
\hline 2 & Nivel de desarrollo bajo & $\begin{array}{l}\text { La empresa tiene dentro de su planeación realizar acciones o programas que contengan } \\
\text { esta práctica, pero no ha pasado a nivel de ejecución. }\end{array}$ \\
\hline 3 & Nivel de desarrollo medio & La empresa ha ejecutado algunas acciones o programas que responden a esta práctica. \\
\hline 5 & Nivel de desarrollo alto & $\begin{array}{l}\text { Los programas y acciones ejecutados en relación a esta práctica son medidos con } \\
\text { indicadores de gestión. }\end{array}$ \\
\hline N/A & No aplica & $\begin{array}{l}\text { Los programas y acciones emprendidos por la empresa se encuentran en una etapa de } \\
\text { mejora e institucionalizados en la empresa. }\end{array}$ \\
\hline
\end{tabular}

Fuente: elaboración propia.

Finalmente, se consideraron 5 niveles de madurez y se incluyó la categoría de no aplica, para no afectar la puntuación de acuerdo a los factores excluyentes a la institución, como se puede apreciar en la tabla.

Además, se incluyó una columna de observaciones con el propósito de recopilar apreciaciones frente a cada uno de los criterios evaluados, tanto en las materias fundamentales como en los principios de la RS.

Para la selección de la muestra se consideró la técnica de muestreo aleatorio estratificado, con el ánimo de asegurar la participación de las diferentes partes interesadas de la IPS objeto de estudio, como se muestra a continuación: 
Tabla 2. Distribución de la muestra por partes interesadas.

\begin{tabular}{|l|c|c|}
\hline $\begin{array}{c}\text { Partes interesadas de } \\
\text { la IPS }\end{array}$ & $\begin{array}{c}\text { Universo } \\
\text { (número total } \\
\text { de personas) }\end{array}$ & $\begin{array}{c}\text { Muestra } \\
\text { seleccionada } \\
\text { (personas } \\
\text { vinculadas en el } \\
\text { estudio) }\end{array}$ \\
\hline Junta & 6 & 4 \\
\hline Usuarios y familias & 10 & 7 \\
\hline Colaboradores & 850 & 123 \\
\hline Acreedores & 4 & 2 \\
\hline Organismo de control & 1 & 1 \\
\hline Proveedores & 30 & 22 \\
\hline Comunidad entorno & 6 & 5 \\
\hline Total & 907 & 164 \\
\hline
\end{tabular}

Fuente: elaboración propia.

Una vez identificados los resultados del diagnóstico de las prácticas de la RS, estos fueron priorizados considerando las materias fundamentales y los principios de la RS con más baja puntuación. Con base en la revisión de literatura y el marco teórico se extractaron estrategias de RS aplicables a la IPS.

Adicionalmente, se efectuó una lluvia de ideas entre el equipo de investigadores y se definieron las mejores alternativas de intervención, considerando tanto la revisión de la literatura como el conocimiento y la amplia experiencia del equipo investigador en el sector salud. A partir de la triangulación de la información mencionada se elaboró una propuesta de metodología para el desarrollo de la RS basada en la norma ISO 26000:2010 - Guía de Responsabilidad Social y en el informe de sostenibilidad GRI-4.

\section{RESULTADOS Y DISCUSIÓN}

En primer lugar, se describen los hallazgos del diagnóstico de las prácticas de RS en la IPS objeto de investigación, tanto de forma global como detallada por cada una de las materias fundamentales y principios de la RS. Se segmentó la muestra por grupos de interés para así poder identificar las percepciones de cada uno de ellos y establecer comparaciones y similitudes.

En segundo lugar, se presenta la propuesta metodológica para el desarrollo de la RS para la IPS objeto de investigación, la cual se basa en los hallazgos del diagnóstico, la revisión de la literatura descrita en el marco teórico, entre las fuentes se encuentra el informe de sostenibilidad GRI y la lluvia de ideas generada por el equipo investigador.

\section{Diaǵnóstico de las prácticas de la Responsabilidad Social en la IPS estudiada}

Para el diagnóstico situacional se abordó una muestra de 164 personas ( $n=164$ ) que hacen parte de los diferentes grupos de interés de la IPS en estudio, de acuerdo con la distribución señalada en la tabla 2.

\section{Análisis detallado de la Responsabilidad Social de acuerdo con las materias fundamentales.}

En la encuesta se evaluaron los siguientes aspectos: 1) gobernanza de la organización; 2) derechos humanos (DDHH); 3) prácticas laborales; 4) medio ambiente; 5) prácticas justas de operación; 6) asuntos de consumidores; 7) participación activa; 7) desarrollo de la comunidad. 
Tabla 3. Resultados por materias fundamentales de la RS.

\begin{tabular}{|c|c|c|c|c|c|c|c|c|}
\hline Grupos de interés & 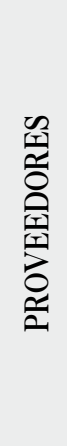 & 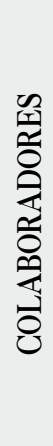 & 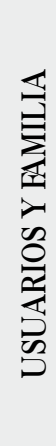 & $\begin{array}{l}0 \\
0 \\
0 \\
0 \\
0 \\
0 \\
0 \\
0 \\
0 \\
0 \\
0 \\
0 \\
0 \\
0 \\
0 \\
0\end{array}$ & 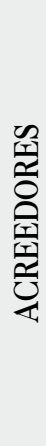 & 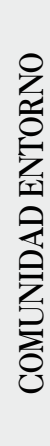 & 吕 & 랭 \\
\hline Gobernanza de la organización & 3.90 & 4.5 & 3.6 & 4 & 3.8 & 3.9 & 3.4 & 3.87 \\
\hline Derechos humanos (DDHH) & 3.89 & 4.3 & 3.9 & 4 & 3.3 & 3.6 & 3.2 & 3.74 \\
\hline Prácticas laborales & 3.86 & 4.2 & 4.1 & 3.2 & 4 & 3.7 & 3.25 & 3.76 \\
\hline El medio ambiente & 3.47 & 4.3 & 3.2 & 1.8 & 3.1 & 3.8 & 2.5 & 3.17 \\
\hline Prácticas justas de operación & 3.77 & 4.3 & 3.6 & 4.5 & 3.6 & 3.7 & 3.4 & 3.84 \\
\hline Asuntos de consumidores & 3.96 & 4.4 & 3.9 & 4 & 3.6 & 4 & 3.7 & 3.94 \\
\hline Participación activa y desarrollo de la comunidad & 3.34 & 4 & 3.3 & 1.1 & 2.4 & 3.3 & 3.1 & 2.93 \\
\hline
\end{tabular}

Fuente: elaboración propia.

Para esta valoración que asignaron los grupos de interés se identificó que estos perciben que las materias fundamentales se encuentran en un nivel de desarrollo medio. Es decir, la IPS ha ejecutado algunas acciones o programas que responden a la práctica de la materia en la gestión cotidiana de la institución. Sin embargo, aún no se encuentran formalmente establecidos ni se han definido mecanismos de medición y seguimiento de la gestión.

A continuación se hará una descripción de las materias fundamentales para su mejor entendimiento:

1. Gobernanza de la organización. En esta materia la percepción de los stakeholders evidencia que la IPS en estudio presenta deficiencias en la estructura o proceso para la toma de decisiones estratégicas; también se denota que los valores de la institución son reconocidos y orientan el cumplimiento de su visión y su misión.
2. Derechos humanos (DDHH). La percepción de los stakeholders evidencia que la IPS en estudio presenta deficiencias en la promoción de trabajo con grupos vulnerables, así como en el establecimiento de mecanismos para solución de conflictos con sus grupos de interés. De igual forma se destaca que esta defiende los derechos laborales y previene los posibles impactos de su operación sobre los derechos humanos.

3. Prácticas laborales. En relación con la gestión del talento humano, los stakeholders manifiestan que la IPS en estudio presenta deficiencias en la implementación de mecanismos de negociación con sus colaboradores, pero se destaca que vela por minimizar los riesgos en seguridad y salud en el trabajo durante su operación diaria.

4. El medio ambiente. La percepción de los stakeholders evidencia que la IPS en estudio no cuenta con los 
procedimientos y medidas necesarias para promover la preservación del medio ambiente en el desarrollo de sus operaciones.

5. Prácticas justas de operación. En esta materia el conocimiento que tienen los stakeholders muestra que la IPS en estudio despliega buenas prácticas en el desarrollo de sus operaciones, aunque podría influir más en la adopción de estas por parte de sus proveedores.

6. Asuntos de consumidores. Los stakeholders manifiestan que la IPS en estudio se esfuerza por generar un buen nivel de servicio a través de diferentes procesos y procedimientos, el cual se refleja en la satisfacción de sus usuarios y demás grupos de interés.

\section{Participación activa y desarrollo de la comunidad.} Respecto a este asunto puede señalarse que la IPS en estudio no presenta una interacción constante y estrecha con la comunidad, por lo que debe implementar acciones que contribuyan con el bienestar de la comunidad de influencia directa.

En una visión general de los resultados obtenidos en la valoración de las materias, se hace manifiesto que los grupos de interés aprecian que las materias fundamentales con un nivel de desarrollo bajo son: medio ambiente, participación activa y desarrollo de la comunidad. Aunque la organización tiene planeado realizar acciones o programas que contengan estas prácticas, las partes interesadas analizadas aún no llevan a la concreta realización de esas materias fundamentales.

A continuación se presenta un análisis más detallado de los asuntos identificados en las materias de medio ambiente y participación activa y desarrollo de la comunidad, por ser las de más baja calificación:

a. En la norma ISO 26000:2010 - Guía de Responsabilidad Social, la gobernanza de la organización ha sido definida como un "sistema por el cual una organización toma e implementa decisiones con el fin de lograr sus objetivos" (Instituto Colombiano de Normalización y Certificación, Icontec, 2010).

b. En medio ambiente se indagó el conocimiento que tienen los grupos de interés respecto a procedimientos que tiene la IPS en estudio para prevenir la contaminación del ambiente y las medidas para reducir consumo de energía, agua y otros recursos.

c. En la materia fundamental participación activa y desarrollo de la comunidad se valoró la percepción de los grupos de interés frente a la participación de la comunidad para determinar actividades de desarrollo y posibles inversiones sociales.

d. En relación al análisis de las materias discriminando los valores por grupos de interés, se señala que la percepción de la junta directiva, organismos de control y acreedores respecto a la gestión de estos asuntos es menor. Se identificó en la aplicación de las herramientas que estos grupos de interés no tienen mayor conocimiento de las prácticas que realiza la institución en términos de mitigación de los impactos negativos en el medio ambiente e interacción con la comunidad.

\section{Análisis detallado de la Responsabilidad Social de acuerdo con los principios.}

Los principios como bloque orientador de la RS en la gestión de la IPS en estudio corresponden a los siguientes aspectos: 1) rendición de cuentas; 2) transparencia, 3) comportamiento ético; 4) partes interesadas; 5) legalidad; 6) normativa internacional; 7) respeto a los derechos humanos. En la tabla 4 se aprecian los hallazgos de acuerdo con los grupos de interés abordados. Comparativamente con las materias fundamentales, estos principios fueron mejor valorados. 
Tabla 4. Resultados de los principios de la Responsabilidad Social.

\begin{tabular}{|c|c|c|c|c|c|c|c|c|}
\hline Grupos de interés & 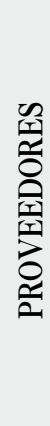 & 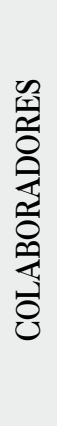 & 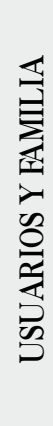 & 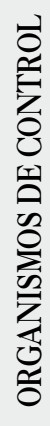 & 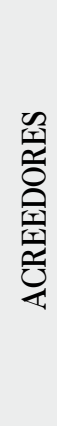 & 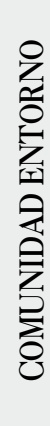 & 壳 & 깅 \\
\hline Rendición de cuentas & 3.9 & 3.9 & 3.8 & 3.8 & 2.3 & 3.7 & 3.8 & 3.6 \\
\hline Transparencia & 4.0 & 4.3 & 4.0 & 4.7 & 4.2 & 3.8 & 3.6 & 4.1 \\
\hline Comportamiento ético & 4.0 & 4.3 & 4.3 & 3.8 & 3.8 & 3.9 & 3.5 & 3.9 \\
\hline Partes interesadas & 3.8 & 4.2 & 4.2 & 4.2 & 3.2 & 3.7 & 3.2 & 3.8 \\
\hline Legalidad & 3.9 & 4.3 & 4.2 & 5.0 & 4.4 & 4.0 & 3.8 & 4.2 \\
\hline Normativa internacional & 4.1 & 4.1 & 3.8 & 5.0 & 1.0 & 3.8 & 3.5 & 3.6 \\
\hline Respeto a los derechos humanos & 3.9 & 4.3 & 4.0 & 4.7 & 4.1 & 3.9 & 3.9 & 4.1 \\
\hline
\end{tabular}

Fuente: elaboración propia.

1. Rendición de cuentas. Sobre este principio la percepción de los stakeholders evidencia que la IPS en estudio cumple con la presentación de informes sobre el desarrollo de actividades relacionadas con RSE.

2. Transparencia. En este principio los stakeholders reconocen que en la IPS en estudio se desarrollan las actividades con total transparencia y ética en pro del bienestar común.

3. Comportamiento ético. Para este principio la percepción de los stakeholders evidencia que la IPS en estudio desarrolla sus actividades y promueve las acciones éticas en el proceder de los diferentes componentes de la organización.

4. Respeto a los intereses de las partes interesadas. Sobre este principio la percepción de los stakeholders evidencia que la IPS en estudio promueve el cumplimiento de sus intereses y de las partes interesadas en el desarrollo de sus operaciones.
5. Respeto al principio de legalidad. Los stakeholders están de acuerdo en que la IPS en estudio cumple con todos los requisitos legales para su operación.

6. Respeto a la normativa internacional de comportamiento. En este principio la percepción de los stakeholders evidencia que la IPS cumple con la normatividad requerida.

7. Respeto a los derechos humanos. En lo que concierne a este principio, la concepción de los stakeholders manifiesta que la IPS en estudio respeta y promueve el acato por los derechos humanos, así como las leyes y normativas correspondientes.

En conclusión, los principios con calificaciones más bajas correspondieron al cumplimiento de la normatividad internacional y rendición de cuentas, esto al parecer se debe a mecanismos de divulgación insuficientes e incluso nulos respecto a los aspectos anteriormente mencionados. 
En el principio de respeto a la normativa internacional se escrutó el conocimiento de los grupos de interés respecto al cumplimiento que tiene la IPS en el estudio de normas internacionales de comportamiento.

Otro de los principios con una evaluación promedio de nivel de desarrollo bajo es el de rendición de cuentas ante la sociedad. En este aspecto se evaluó si la organización responde a los escrutinios realizados por las partes interesadas frente a la gestión de la $\mathrm{RS}$ en el componente económico, y si la dirección de la organización entrega informes de acuerdo con las regulaciones relacionados con la gestión de la RS.

A partir del procesamiento de los datos, se encontró que los grupos de interés que valoraron con una menor calificación los principios fueron: los acreedores y la junta directiva. Se identifica que la IPS en estudio no tiene un plan de involucramiento establecido, por lo cual a los grupos de interés que no se encuentran directamente relacionados con la gestión propia de la institución, no se les comunican los resultados de la operación.

En un análisis global de los resultados se identificó que el $37 \%$ de los encuestados realizó observaciones como complemento de sus respuestas; los ítems en los que se identificó que un mayor número de personas manifestó no tener conocimientos. Cabe acotar que no se obtuvo información de orden cualitativa derivada de los comentarios de los participantes del estudio que requiriera otro tipo de procesamiento. En otras palabras, las observaciones de los participantes se limitaron a tres apreciaciones "estoy de acuerdo", "no conozco, no sé al respecto lo que la institución realiza" o "falta comunicación".

Al consolidar los resultados obtenidos sobre el desempeño de la IPS en estudio en cada una de las materias analizadas, se evidenció que en promedio es aceptable, por lo que se deben generar actividades enfocadas al fortalecimiento de estas. Por otro lado, al analizar el resultado obtenido sobre los principios, se denota un desempeño más alto, aunque también debe reforzarse para obtener mejores resultados en torno a sus stakeholders, en especial lo que corresponde al relacionamiento con sus acreedores.

\section{Elementos de una metodología para el desarrollo de la Responsabilidad Social en la IPS de tercer nivel de complejidad}

Acorde con los resultados obtenidos del diagnóstico y a la realidad conocida, se planteó la propuesta puntual en un marco metodológico que es el fin principal de la investigación.

La propuesta que se plantea en este documento, a fin de ser aplicado en instituciones de salud como es la IPS en estudio de tercer nivel de complejidad en la ciudad de Bogotá, tiene sus bases en los lineamientos que se desarrollan en cada uno de los apartes de la norma ISO 26000:2010 - Guía de Responsabilidad Social, con un especial énfasis en lo estipulado en relación con la integración de la RS en toda la organización.

La guía da las directrices a seguir, las cuales se orientan a establecer la manera en que las características de la organización se relacionan con la RS, para luego entrar a entender y comprender la RS de la institución identificando los impactos sociales, ambientales y económicos, determinando la pertinencia e importancia de las materias fundamentales y principios definidos en esta norma.

\section{Enfoques y etapas de la metodología de la Responsabilidad Social}

La norma ISO 26000:2010 - Guía de Responsabilidad Social, enuncia tres enfoques principales que se deben tener en cuenta en la RS: enfoque social, ambiental y económico. Para que la IPS logre vincular la RS en su 
institución requiere de la incorporación de estos tres enfoques en su direccionamiento estratégico y gestión diaria y transversal de la institución (nivel estratégico, táctico y operativo).
Adicionalmente, a partir de los hallazgos derivados de la revisión de la literatura y del diagnóstico situacional, se evidencia la necesidad de cumplir con seis etapas de la RS como se detalla en la figura 2.

Figura 1. Responsabilidad Social en la IPS.

Equilibrio socioambiental y económico de la institución

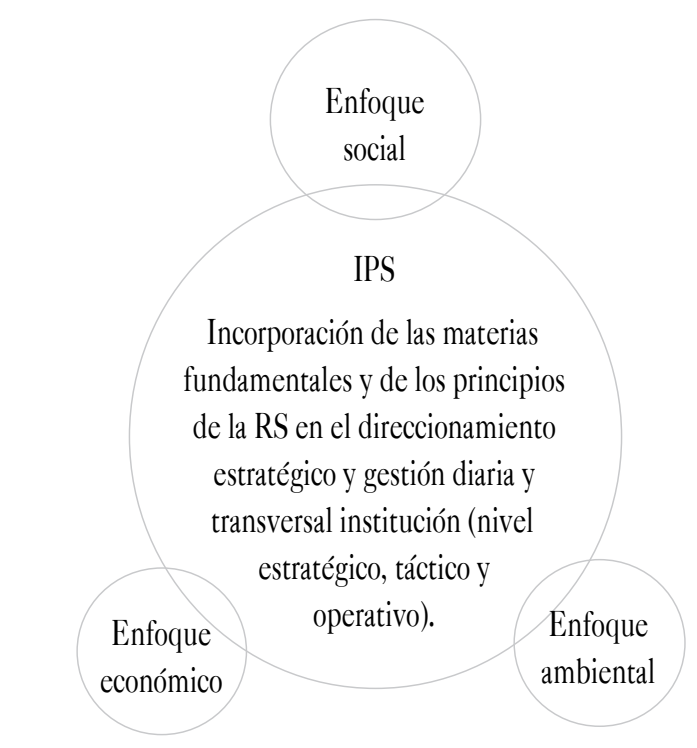

Fuente: elaboración propia.

Figura 2. Propuesta metodológica de aplicación de la Responsabilidad Social.

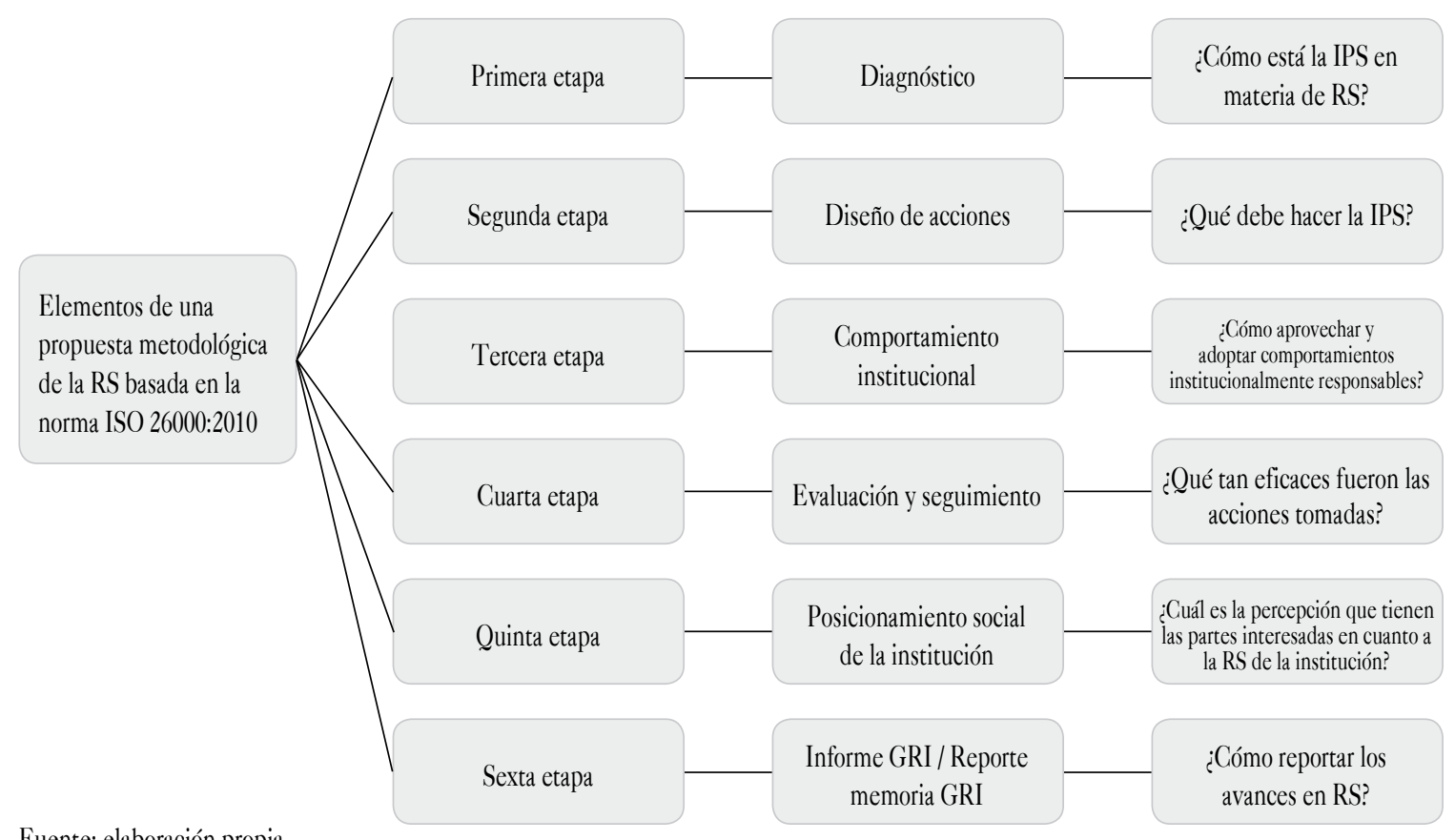

Fuente: elaboración propia. 


\section{Primera etapa: diagnóstico de la Responsabilidad Social en la IPS.}

La IPS en estudio debe identificar y entender la RS, por ende, es necesario realizar un diagnóstico situacional de la organización de acuerdo con los principios y materias fundamentales de RS contemplados en la norma ISO 26000:2010 - Guía de Responsabilidad Social. La metodología que se propone busca reconocer los grupos de interés que están relacionados con los procesos y el plan estratégico de la organización, ya que en cada actividad los grupos de interés están involucrados con la IPS.
Para la realización del diagnóstico situacional, la IPS podráhacer uso de la herramienta que se diseñó de acuerdo con los siete principios y siete materias fundamentales de la norma ISO 26000:2010 - Guía de Responsabilidad Social (ver figura 3 y 4 ) dado que su escala le permite evaluarse transversal y periódicamente y hacer comparativos de los hallazgos pre y posintervenciones o procesos de mejora de su gestión en RS. Para el desarrollo del diagnóstico se puede hacer uso como complemento de otras herramientas como el DOFA, el Pesta, las cinco fuerzas de Porter, entre otras.

Figura 3. Siete principios de la Responsabilidad Social.

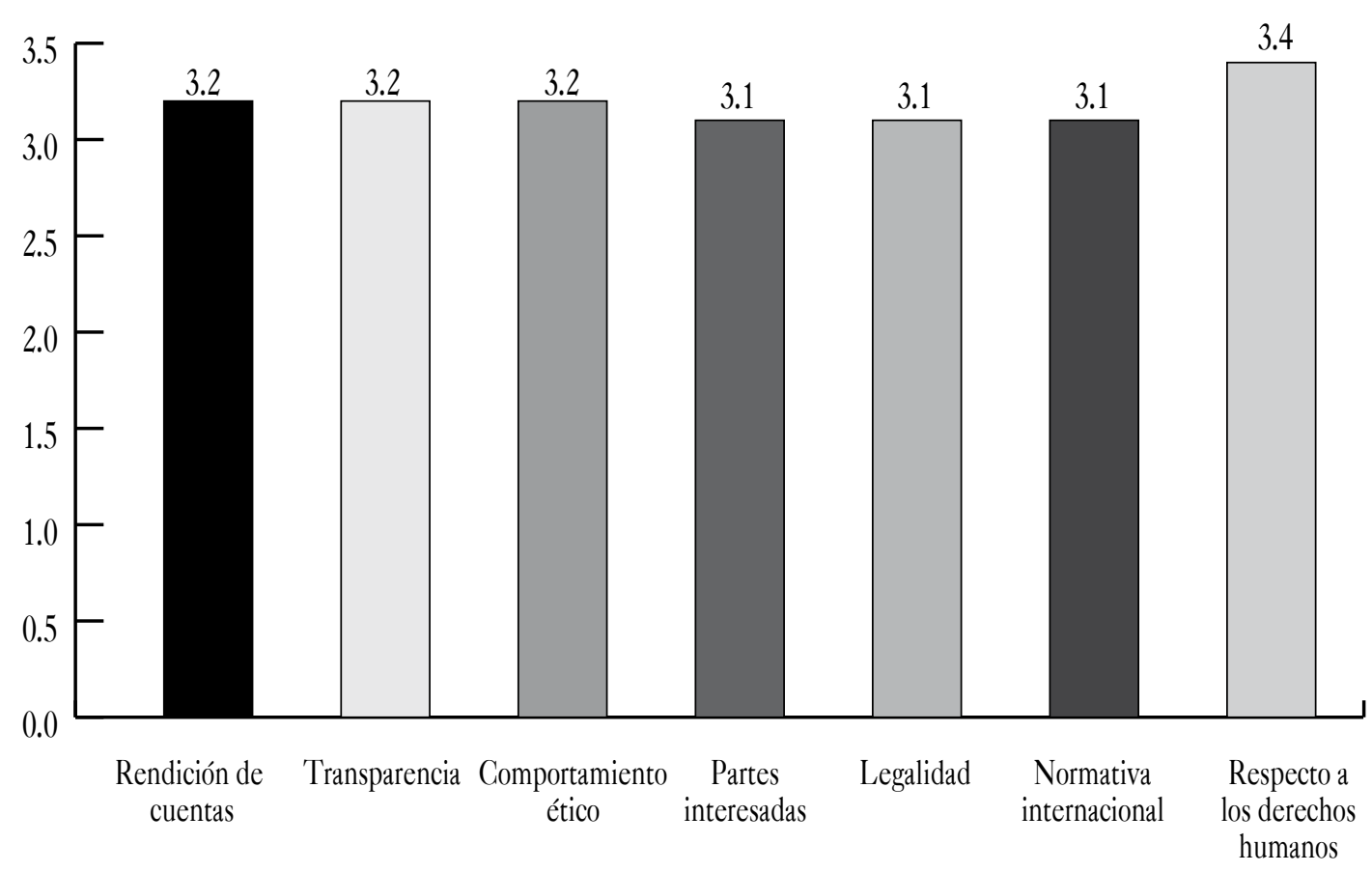

Fuente: elaboración propia. 
Figura 4. Siete materias fundamentales de la Responsabilidad Social.

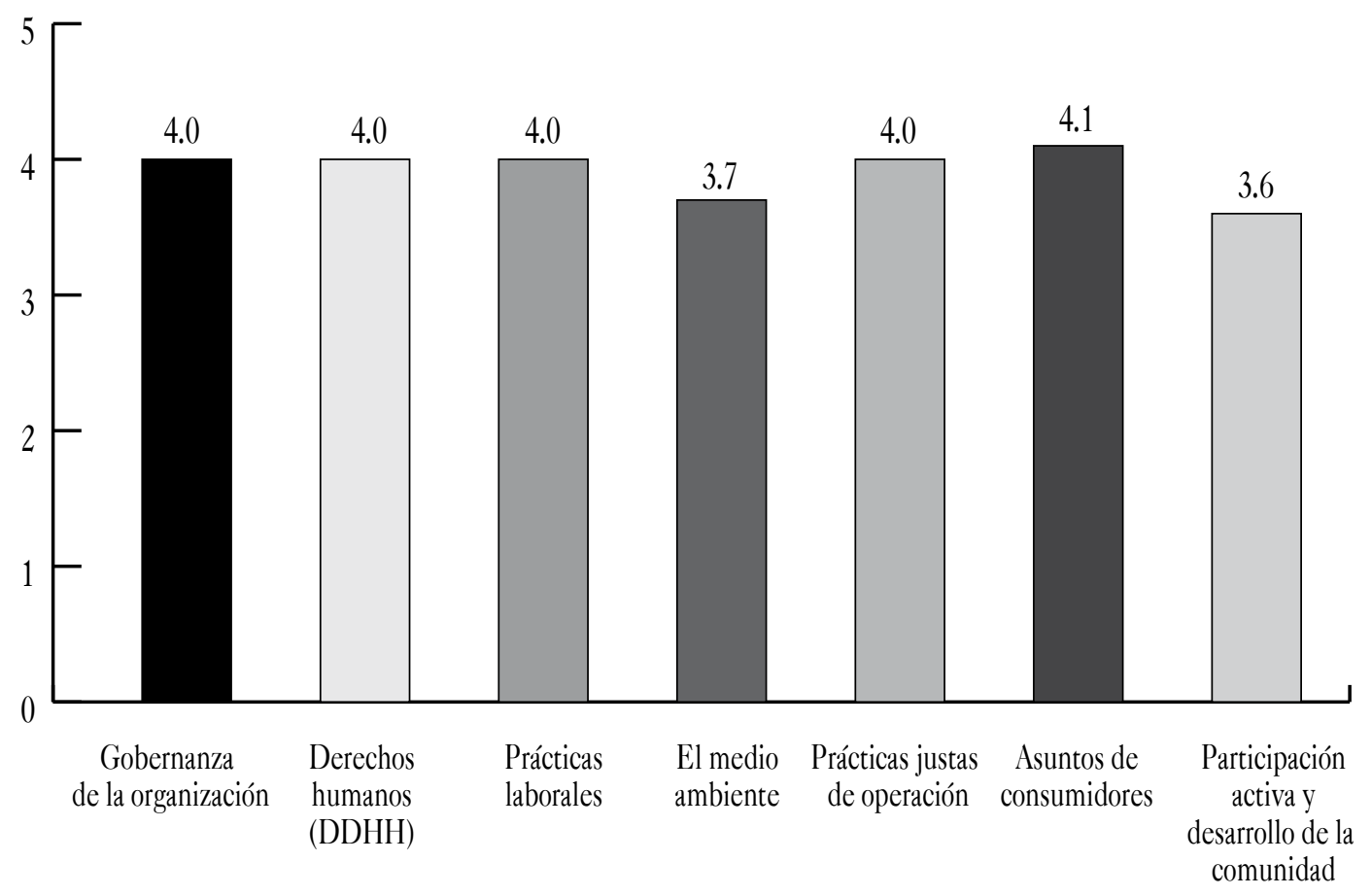

Fuente: elaboración propia.

El resultado del diagnóstico permitirá a la IPS priorizar dos o tres materias fundamentales y principios en las cuales debe generar foco de intervención para así poder mejorar sus prácticas en RS. Esta priorización se define con los resultados globales de la puntuación, es decir, se deben elegir las dos materias fundamentales y los principios con más baja puntuación.

Particularmente en la IPS objeto de investigación se priorizaron dos materias fundamentales (medio ambiente y participación activa y desarrollo de la comunidad, por ser las de más baja calificación) y dos principios (cumplimiento de la normatividad internacional y rendición de cuentas), debido a que correspondieron a los aspectos con más baja calificación de acuerdo con la percepción de las partes interesadas. Sobre estos aspectos se plantearon la mayoría de las acciones de mejora presentadas en la segunda etapa.

\section{Segunda etapa: diseño de acciones socialmente responsables.}

A partir del diagnóstico se identifican los materias y principios de la norma ISO 26000:2010 - Guía de Responsabilidad Social, en los cuales la IPS tiene mayores oportunidades de mejora para implementar acciones y estrategias de RS que sean posibles de ejecutar y desplegar en los diferentes procesos institucionales.

Para el caso de la IPS estudiada, como se mencionó antes las materias fundamentales que de manera prioritaria se deben intervenir fueron: participación activa y desarrollo de la comunidad, con una calificación de 2.93 sobre 5; medio ambiente con una calificación de 3.17; normatividad internacional con una calificación de 3.6 sobre 5 ; rendición de cuentas con un 3.6 sobre 5 . Aunque no son 
calificaciones del todo bajas, sí reflejan la oportunidad de mejora en la integración de la responsabilidad.

En tal sentido, esta etapa es relevante toda vez que de ella depende el diseño de forma articulada con el plan estratégico de las estrategias de buenas prácticas para la implementación y desarrollo de la RS en la IPS. La decisión de la ruta estratégica implica establecer la articulación y la formulación de indicadores que permitan la sinergia entre la planeación estratégica de la organización y las intenciones acerca de la RS, permitiendo formalmente ver los resultados del compromiso de la empresa respecto a la RS.

A partir de los resultados obtenidos del diagnóstico de la RS en la IPS, descrito en el apartado anterior, así como a partir del consenso con los grupos de interés de la IPS, se proponen las siguientes acciones:

Tabla 5. Acciones de intervención.

\begin{tabular}{|c|c|c|}
\hline Grupo de interés & Objetivo & Mecanismos de relacionamiento \\
\hline Junta directiva & $\begin{array}{l}\text { Fomentar un ambiente de confianza entre } \\
\text { la junta y la alta dirección para definir } \\
\text { políticas orientadas a la sostenibilidad } \\
\text { de la institución, basadas en información } \\
\text { objetiva y oportuna. }\end{array}$ & $\begin{array}{l}\text { Diálogo y participación } \\
\text { Mesas de trabajo } \\
\text { Boletines } \\
\text { Informes } \\
\text { Correos }\end{array}$ \\
\hline Accionistas & $\begin{array}{l}\text { Promover un ambiente de confianza entre } \\
\text { accionistas y público en general, a través } \\
\text { de una adecuada comunicación trasparente } \\
\text { y oportuna de información relevante } \\
\text { y confiable, con el fin de entregar la } \\
\text { información adecuada para la toma de } \\
\text { decisiones de inversión. }\end{array}$ & $\begin{array}{l}\text { Página web } \\
\text { Boletín trimestral } \\
\text { Correo electrónico } \\
\text { Tele Conferencias } \\
\text { Informe integrado } \\
\text { Asamblea de accionistas } \\
\text { Línea ética }\end{array}$ \\
\hline Colaboradores & $\begin{array}{l}\text { Fomentar relaciones armoniosas para } \\
\text { aumentar la productividad en ambientes } \\
\text { laborales seguros y saludables, en los que } \\
\text { se incentive los resultados y garantice el } \\
\text { desarrollo humano y la calidad de vida en } \\
\text { el trabajo. }\end{array}$ & $\begin{array}{l}\text { Encuetas de clima } \\
\text { Encuestas de riesgo Psicosocial } \\
\text { organizacional } \\
\text { Intranet } \\
\text { Boletines } \\
\text { Carteleras } \\
\text { Grupos primarios } \\
\text { Entrevistas de retiro } \\
\text { Comité de convivencia laboral } \\
\text { Informe semestral }\end{array}$ \\
\hline Usuarios y sus familias & $\begin{array}{l}\text { Contribuir a la calidad de vida de los } \\
\text { usuarios por medio de servicios que } \\
\text { satisfagan las necesidades de salud y } \\
\text { calidad de vida a través servicios seguros, } \\
\text { oportunos y humanos, logrando con ello } \\
\text { estar más cerca de los usuarios y sus } \\
\text { familias. }\end{array}$ & $\begin{array}{l}\text { Figuras comerciales que interactúan con los } \\
\text { pacientes y sus familias } \\
\text { Portal del cliente (pacientes y familias) } \\
\text { Línea de atención } \\
\text { Correo electrónico } \\
\text { Informes }\end{array}$ \\
\hline Comunidad y su entorno & $\begin{array}{l}\text { Asegurar el desarrollo de capacidades } \\
\text { al interior de las comunidades como } \\
\text { herramienta para alcanzar su sostenibilidad } \\
\text { y apoyar el bienestar por medio de la } \\
\text { destinación de recursos tangibles e } \\
\text { intangibles. }\end{array}$ & $\begin{array}{l}\text { Reuniones de encuentro } \\
\text { Capacitaciones en temas ambientales } \\
\text { Eventos } \\
\text { Participación en foros } \\
\text { Proyección social } \\
\text { Participación Mesa de Trabajo Gestión } \\
\text { Ambiental Pacto Global }\end{array}$ \\
\hline
\end{tabular}


Hermelinda Gómez Ramírez, Eliana Carolina Herrera Pérez, Ligia Edilma Sorro Corredor

\begin{tabular}{|c|c|c|}
\hline Grupo de interés & Objetivo & Mecanismos de relacionamiento \\
\hline Estado & $\begin{array}{l}\text { Fomentar la participación de } \\
\text { representantes de la IPS en escenarios } \\
\text { de formulación de política pública que } \\
\text { favorezca los objetivos de la organización y } \\
\text { las necesidades de sus partes interesadas y } \\
\text { de la comunidad en general en materia de } \\
\text { salud y protección social. }\end{array}$ & $\begin{array}{l}\text { Participación en los espacios donde se } \\
\text { promueva el mejoramiento de las políticas } \\
\text { estatales. } \\
\text { Informe y reuniones de control } \\
\text { Diligenciamiento de encuestas } \\
\text { Informe integrado } \\
\text { Participación de Proyectos Hospitales Verdes } \\
\text { Secretaría de Salud. }\end{array}$ \\
\hline Proveedores & $\begin{array}{l}\text { Lograr el fortalecimiento y desarrollo de } \\
\text { las relaciones con los socios en la cadena } \\
\text { de abastecimiento con los proveedores. }\end{array}$ & $\begin{array}{l}\text { Portal de negocio en línea } \\
\text { Participación Proyectos Hospitales Verdes } \\
\text { Secretaría de Salud } \\
\text { Mesa de ayuda } \\
\text { Correo electrónico } \\
\text { Programas para desarrollo de proveedores } \\
\text { Visitas de evaluación } \\
\text { Informes } \\
\text { Implementación uso de papel ecológico - } \\
\text { Direcciones }\end{array}$ \\
\hline
\end{tabular}

Fuente: elaboración propia.

La IPS en estudio tendría varias acciones a realizar orientado a obtener un buen posicionamiento, con el ánimo de hacer realidad la visión que tiene proyectada para el 2020, la de ser una IPS socialmente responsable.

\section{Tercera etapa: implementación de acciones de Responsabilidad Social.}

Esta etapa corresponde a la implementación de las acciones importantes y necesarias para desplegar un plan de intervención creando nuevas acciones y expectativas para la organización, a fin de reducir la brecha obtenida en materia de RS.

Por otra parte, estructurar la políitica de RS de la IPS de estudio de forma integrada en la gestión global de la institución, teniendo en cuenta el plan estratégico y los ejes de acreditación, como la misión y visión de la organización. Así mismo la organización debe determinar los recursos necesarios para la ejecución, teniendo en cuenta el equilibrio económico, social y ambiental.
En esta etapa de implementación la IPS debe sensibilizar y capacitar a los responsables acerca de la norma ISO 26000:2010 - Guía de Responsabilidad Social, para desarrollar las competencias necesarias que permitan el logro y los planes acordes con los objetivos estratégicos planteados.

La IPS deberá ejecutar las acciones previstas en la etapa anterior de acuerdo con la planeación efectuada y el cronograma de trabajo establecido para ello.

\section{Cuarta etapa: evaluación y seguimiento.}

En la cuarta etapa, es importante realizar una evaluación constante de las actividades implementadas, que permitan identificar las mejoras en RS y analizar la operación y los resultados económicos, sociales y ambientales de la organización.

El modelo de gestión de la RS permite ver los resultados de un buen desarrollo para encontrar beneficios en términos económicos, sociales y ambientales, los cuales son el efecto lógico de una buena gestión, donde se 
Figura 5. Requisitos de la Responsabilidad Social.

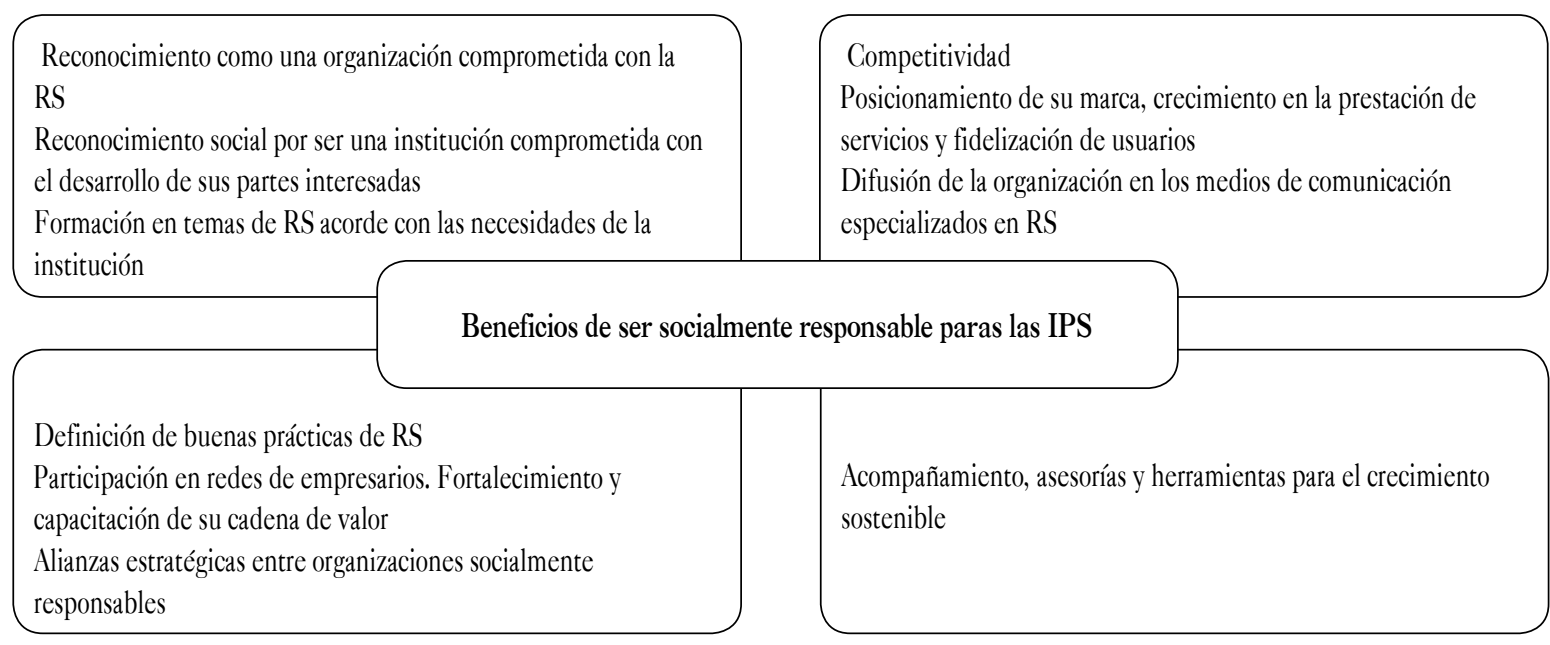

Fuente: elaboración propia.

puede reconocer las bondades que se contemplan de ser una empresa socialmente responsable.

En este punto la IPS deberá acoger los indicadores de desempeño de RS descritos en el GRI, acordes con las necesidades identificadas por los grupos de interés.

\section{Quinta etapa: posicionamiento social de la institución.}

La RS requiere de políticas sustentadas en fundamentos éticos, valores y principios, donde las partes se beneficien mutuamente mediante herramientas objetivas y transparentes.

Una vez la gestión de la RS esté debidamente estructurada y posicionada, la organización podrá aspirar a la obtención de reconocimientos de carácter nacional e internacional, a saber: autoevaluación por parte de Fenalco, adhesión al programa Pacto Global, sello colombiano ambiental, certificación en ISO 14001, hospitales verdes liderados por la Secretaria Distrital de Salud.

Una organización (IPS) que asume la RS es aquella que cumple, se compromete y tiene en cuenta los siguientes puntos: 1) incluye objetivos sociales en sus procesos de planeación; 2) aplica en sus programas sociales normas comparables a las de las otras organizaciones; 3) presenta informes a los miembros de la organización y a los grupos de interés sobre los avances de su responsabilidad social; 4) experimenta con distintos enfoques para medir su desempeño social; 5) trata de medir los costos de los programas sociales y el rendimiento de las inversiones en acciones sociales.

La IPS podrá revisar experiencias significativas en materia de RS en Colombia, como son la Fundación Cardiovascular de Colombia y el Hospital Pablo Tobón Uribe en Medellín. Esto con el ánimo de identificar prácticas innovadoras de RS como complemento a la información recopilada a través del diagnóstico de RS en la IPS, efectuado en la etapa 1 de esta metodología.

\section{Sexta etapa: guía para la elaboración de memorias de sostenibilidad, informe GRI-4.}

La guía para la elaboración de memorias de sostenibilidad, informe GRI- 4, constituye una referencia internacional para aquellos interesados en la información 
sobre el enfoque de gestión y el desempeño y la repercusión de las organizaciones en los ámbitos ambiental, social y económico, los cuales son la base de la RS.

Esta guía es un referente que permite mayor objetividad en la presentación de los avances de la RS en las organizaciones, además da parámetros globales que pueden ser comparables con otras organizaciones, aspecto que le permitiría a la institución medirse frente a referentes externos nacionales e internacionales. Estos requisitos son aplicables a cualquier organización independientemente de su tamaño. Por consiguiente, con el fin de garantizar una presentación equilibrada y razonable del desempeño de la organización, se debe determinar el contenido que debe incluir la memoria. Esto debe hacerse teniendo en cuenta tanto la experiencia y el propósito de la organización, como los intereses de sus grupos interés. Ambos puntos de referencia son importantes a la hora de decidir qué debe incluirse en la memoria.

Se recomienda a la IPS elaborar este informe de forma anual para así ajustar el plan de acciones de RS, así como los objetivos y la política con base en los hallazgos de este informe y los resultados de la etapa 1 de la metodología. En la figura 6, se presenta la estructura global de las etapas y de los aspectos que se deben considerar en el informe GRI -4.

Figura 6. Estructura global del informe.

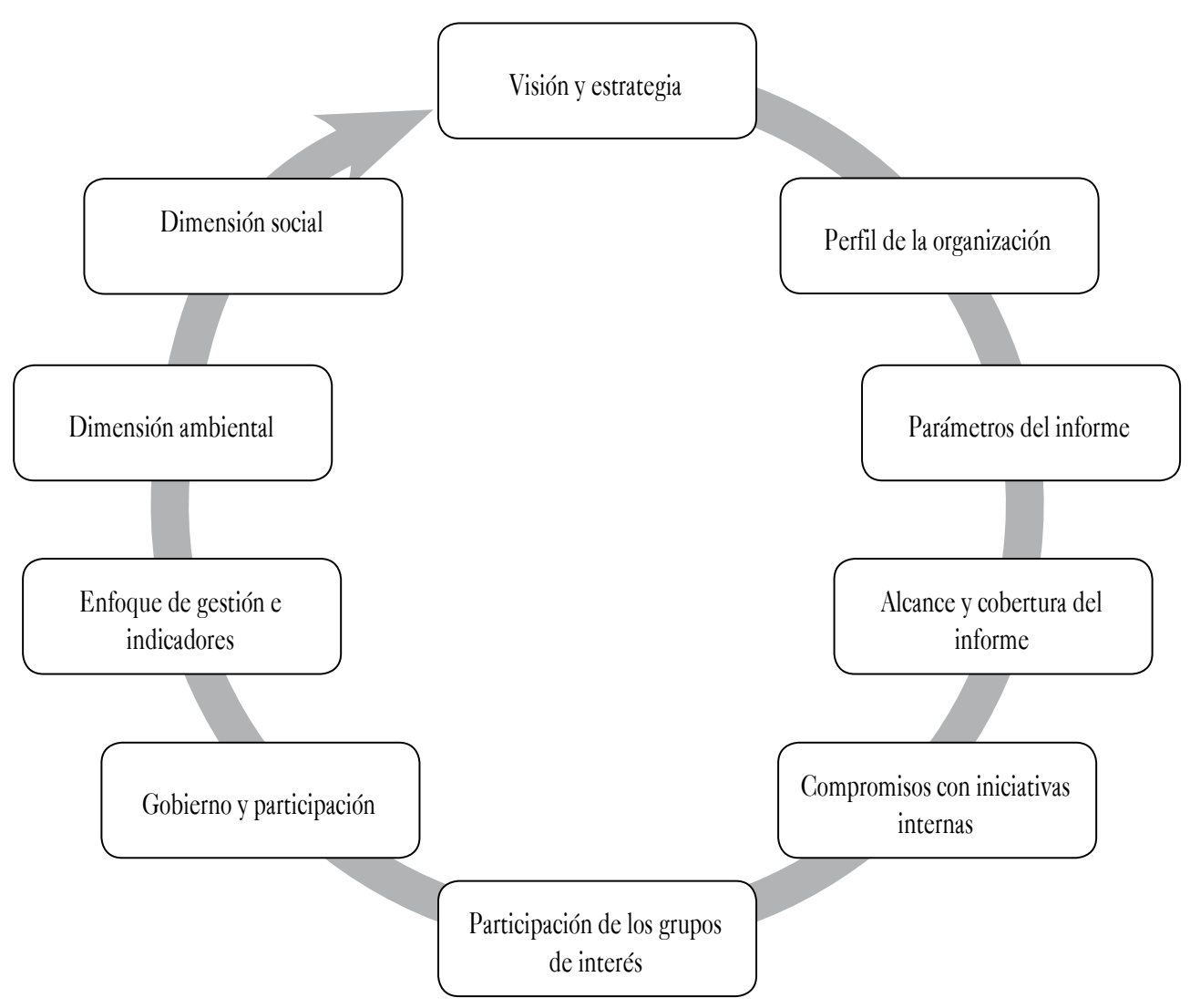

Fuente: elaboración propia. 
Finalmente, este informe le permitirá a la IPS presentar de forma sintética, clara y precisa sus prácticas en RS, así como los avances en su gestión a las distintas partes interesadas. Esto como respuesta a su necesidad de crear sinergia entre los grupos de interés con la institución, propendiendo con ello por la generación de servicios de salud con calidad y humanizados, así como al desarrollo sostenible de la IPS.

\section{CONCLUSIONES}

La investigación adelantada permitió determinar los elementos de una metodología para el desarrollo de la RS basada en los requisitos de la norma ISO 26000:2010 - Guía de Responsabilidad Social dirigida a la IPS y el estado actual de desarrollo de la RS en una IPS de tercer nivel de complejidad en la ciudad de Bogotá.

Esta metodología se estructuró en seis etapas que incluyen el diagnóstico de la RS, el diseño de las acciones de intervención con base en los hallazgos de la etapa anterior, el comportamiento institucional, la evaluación y el seguimiento de las acciones de intervención en RS, el posicionamiento de la institución y el informe GRI o reporte de memoria GRI que da cuenta de los avances de la IPS en RS.

Por otra parte, se desarrolló un instrumento para el diagnóstico de las prácticas de RS en IPS, el cual cuenta con validez de contenido generada a partir de su revisión técnica y de la aplicación de la prueba piloto. Este instrumento es una herramienta que puede ser usada tanto por la IPS estudiada como por otras IPS con características similares, para la evaluación de las prácticas de RS.

Asimismo, se logró articular los principios y materias fundamentales de la Responsabilidad Social de la norma ISO 26000:2010 - Guía de Responsabilidad Social con el informe GRI para una IPS de tercer nivel de complejidad de la ciudad de Bogotá, los cuales son dos referentes internacionales que aportan en el diseño, implementación, reporte y mejora de las prácticas en $\mathrm{RS}$ en las organizaciones en general.

Además, se concluyó que la RS aporta a las organizaciones un marco referencial creativo en tres ámbitos esenciales para su desarrollo, a saber: económico, social y ambiental. Cabe precisar, que la adopción de estándares de gestión de la RS facilita el cumplimiento de los requisitos del SOGCS establecido para las IPS colombianas, adicionalmente fomentan una cultura de $\mathrm{RS}$ en estas instituciones, lo que a su vez contribuye en la prestación de servicios de calidad y humanizados.

Para finalizar, se evidenció que el desarrollo de la RS es de gran valor para la IPS estudiada, por su contribución en el cumplimiento de los requisitos del Sistema Único de Acreditación en Salud, en la mejora de la imagen y en el reconocimiento de la IPS en el sector y en la comunidad en la cual está inmersa.

A partir de los resultados de la presente investigación se emiten las siguientes recomendaciones dirigidas a académicos e investigadores en el campo de los sistemas de gestión en RS: 1) validar el constructo de la propuesta de metodología para el desarrollo de la responsabilidad basada en los requisitos de la norma ISO 26000:2010 - Guía de Responsabilidad Social en otras IPS de diferentes niveles de complejidad. De igual manera esta propuesta podría probarse en otros sectores económicos; 2) desarrollar nuevas investigaciones que incorporen los requisitos de la norma ISO 26000:2010 - Guía de Responsabilidad Social y el informe GRI, de tal manera que sus datos puedan ser comparados con investigaciones en otros sectores, tanto en el ámbito nacional como en el internacional; 3) validar el constructo del instrumento propuesto para el diagnóstico de las prácticas de RS en IPS, el cual cuenta con validez de contenido; 4) utilizar las herramientas desarrolladas en esta investigación (propuesta 
de metodología para el desarrollo de la responsabilidad basada en los requisitos de la norma ISO 26000:2010 Guía de Responsabilidad Social e instrumento para el diagnóstico de las prácticas de RS en IPS) en procesos de consultoría organizacional, dado que proporcionan información confiable para la toma de decisiones en materia de la organización y el diseño de sistemas de gestión de RS y de gestión integrada.

Finalmente, para la IPS objeto de investigación se recomienda implementar las acciones propuestas para la incorporación de la RS en la gestión diaria y global de la IPS. Asimismo, la institución deberá garantizar los recursos necesarios para la implementación de la propuesta de metodología para el desarrollo de la responsabilidad basada en los requisitos de la norma ISO 26000:2010 Guía de Responsabilidad Social desarrollada con base en las necesidades identificadas por sus partes interesadas.

\section{REFERENCIAS}

Asamblea General de las Naciones Unidas. (s.f.). Desarrollo Sostenible. España. Recuperado de http://www. un.org/es/ga/president/65/issues/sustdev.shtml

Bernal, C. (2010). Metodología de la investigación, administración, economía, humanidades y ciencias sociales. Colombia: Pearson Educación.

Delgado, M., Vásquez, M., y Moraes, L. (2010). Calidad en los servicios de salud desde los marcos de sentido de diferentes actores sociales en Colombia y Brasil. Revista Salud Pública, 12(4), 533-545. Recuperado de https:// www.integrarse.org/organizaciones-miembros/ asociacion-empresarial-para-el-desarrollo-aed

García, I., Vargas., I, Mogollón, A. S., De Paepe, P., Da Silva, M. R., Unger, J. P., \& Vázquez, M. L. (2014). Barriers in access to healthcare in countries with different health systems. A cross-sectional study in municipalities of central Colombia and north-eastern Brazil. Soc Sci Med, 106, 204-213.

Global Reporting Initiative. (2011). GRI e ISO 26000: cómo usar las Directrices del GRI, en conjunto con la Norma ISO 26000. Recuperado de https://www.globalreporting.org/ resourcelibrary/Spanish-GRI-ISO-Linkage-Document-Updated-Version.pdf

González, M. (2005). La responsabilidad social corporativa o responsabilidad social de la empresa. Jornada de economía alternativa y solidaria. Bogotá: Panamericana Editores.

Hernández, R., Collado, F., y Baptista, L.P. (2014).Metodología de la investigación. México: McGraw-Hill.

Instituto Colombiano de Normalización y Certificación. (2010). Guía de Responsabilidad Social. ISO 26000. Bogotá: Icontec.

ONU. (2016). Desarrollo Sostenible. Recuperado de http://www.un.org/es/ga/president/65/issues/ sustdev.shtml

Pérez, M. A. (2012). Conceptualización sobre el Desarrollo Sostenible: operacionalización del concepto para Colombia. Punto de Vista, 3(5), 139-158. Recuperado de https://dialnet.unirioja.es/descarga/ articulo/4776961.pdf

Programa de Naciones Unidas para el Desarrollo - PNUD. (s.f.). Conceptualización sobre el Desarrollo Sostenible: operacionalisación del concepto para Colombia. Recuperado de https://www. un.org/sustainabledevelopment/es/objetivos-de -desarrollo-sostenible/

Vargas, I., Vásquez, M., Mogollón, A. (2010). Barriers of access to care in a managed competition model: lessons from Colombia. BMC Health Serv Res, 10, (pp. 10-297). 